\title{
Progress towards gene therapy for haemophilia B
}

\author{
Nishil Patel · Ulrike Reiss · Andrew M. Davidoff • \\ Amit C. Nathwani
}

Received: 3 January 2014 / Accepted: 13 January 2014/Published online: 6 February 2014

(C) The Japanese Society of Hematology 2014

\begin{abstract}
Haemophilia B is an X-linked recessive bleeding disorder, arising from a deficiency of coagulation factor IX. It has been a target for gene therapy ever since the factor IX gene was cloned in 1982. Several distinct approaches have been evaluated in humans over the last 30 years, but none has resulted in tangible corrections of the bleeding phenotype in humans until recently. Our group has now shown that lasting clinical improvement of the bleeding phenotype in patients with haemophilia $\mathrm{B}$ is possible following a single systemic administration of a self-complementary adeno-associated virus vector to deliver an optimised factor IX expression cassette to the liver. Success in this trial raises hope for patients with severe haemophilia B as well as others with inherited monogenetic disorders of the liver where current treatment options are limited.
\end{abstract}

Keywords Haemophilia · Gene therapy $\cdot$ AAV

N. Patel · A. C. Nathwani $(\bowtie)$

Katharine Dormandy Haemophilia Centre and Thrombosis Unit, Royal Free Hospital, Royal Free NHS Foundation Trust, Pond Street, London NW3 2QG, UK

e-mail: amit.nathwani@ucl.ac.uk

N. Patel · A. C. Nathwani

Department of Haematology, UCL Cancer Institute, London, UK

U. Reiss

Department of Haematology, St. Jude Children's Research Hospital, Memphis, TN, USA

A. M. Davidoff

Department of Surgery, St. Jude Children's Research Hospital, Memphis, TN, USA

A. C. Nathwani

National Health Services Blood and Transplant, London, UK

\section{Introduction}

Haemophilia B, an X-linked bleeding condition has long been recognised as an ideal target for gene therapy approaches. It is a monogenetic disorder that arises because of mutations in the Factor 9 (FIX) gene, which codes for FIX protein, a serine protease that is essential for normal blood clot formation [1]. Therefore, haemophilia B patients suffer from recurrent, often life threatening, bleeding episodes that can occur without any apparent injury. Current treatment consisting of FIX protein concentrate infusion is effective at arresting bleeding episodes, but it is not curative. Prophylaxis with FIX protein concentrates has been shown to reduce the frequency of spontaneous bleeding, but entails intravenous injections of FIX protein every 2-3 days (due to the short half-life of the protein) for the life-time of patients, which is invasive, inconvenient and expensive ( $£ 140,000 /$ year/patient). Partly because of the high cost of factor concentrates, approximately $80 \%$ of haemophilia patients receive no or only sporadic treatment and are condemned to shortened lives of pain and disability [2]. Novel clotting formulations based on pegylated clotting factor proteins or fusion proteins with albumin or $\mathrm{Fc} \gamma$ with longer half-lives do not remove the problems of lifelong intravenous factor administration, break-through bleeding, mounting cost and induction of inhibitory antibodies against protein concentrates. In addition, the consequences of lifelong administration of pegylated proteins are unknown, as is the immunogenic potential of using fusion proteins $[3,4]$.

The rationale for treating haemophilia by a gene transfer approach is well established. First, the coagulation defect results from a single genetic lesion, a point mutation or a more complex mutation, in the gene encoding factor IX. Second, the FIX gene and many of the associated mutations 
have been well characterised. Third, and perhaps the most important, years of clinical experience with protein concentrate infusion indicates that the severe clinical course in patients with plasma FIX levels of less than $1 \%$ of physiologic levels can be significantly ameliorated by a relatively small increase in plasma FIX levels above that level. Therefore, the therapeutic end point for clinical benefit is relatively modest. Despite the fact that haemophilia A is 5 times more common, the early focus has been mainly on haemophilia B, because the FIX cDNA is small enough to fit into most vector systems and has a more efficient cellular processing and secretion profile than human FVIII, the clotting factor deficient in patients with haemophilia A.

Many different vector systems have been evaluated for haemophilia gene therapy, each having strengths and weaknesses [5]. We and others have focused on vectors based on adeno-associated virus (AAV), a single-stranded parvovirus, because of its excellent safety profile. AAV is endemic in the human population, but does not cause disease. Most individuals seroconvert after an infection with AAV with the development and persistence of neutralising antibodies directed against capsid epitopes. A key advantage of AAV vectors is that they mediate expression mainly from episomally retained proviral DNA. AAV vector proviral DNA can integrate into host chromosomal DNA, but at a very low frequency $(0.01 \%$ of cellular DNA is integrated), thus the risk of insertional oncogenesis is low, unlike for retroviral vectors [6]. Importantly, a single administration of AAV vector encoding FIX has resulted in long-term expression of FIX protein at therapeutic levels in murine and canine models of haemophilia B, resulting in a lifetime correction of the bleeding tendency without toxicity [7-9].

It has proven harder to get similar results in patients with severe haemophilia B. Intramuscular administration of AAV2 vectors encoding FIX was safe [10]. Muscle biopsy showed evidence of expression of FIX at the site of administration, but this did not result in sustained plasma FIX levels at $>1 \%$ of physiologic values [11]. A subsequent trial with AAV2 vectors administered into the hepatic artery was briefly effective in one subject of the seven treated. This subject was treated at the highest dose of $2 \times 10^{12} \mathrm{vg} / \mathrm{kg}$ and showed an increase in plasma FIX levels to peak values of $12 \%$ of normal at approximately 4 weeks after gene transfer. Unfortunately, the transduced liver cells appeared to have subsequently been eliminated by a $\mathrm{T}$ cell-mediated immune response to vector capsid. Consequently, the plasma FIX level fell to base line values of $<1 \%$ within 8 weeks of vector infusion [12]. The second subject treated at the high-dose level had a higher neutralising anti-AAV2 antibody titre resulting in effective blockade of hepatocyte transduction following administration of the vector into the hepatic artery. Thus, both humoral and cellular immunity appeared to be significant obstacles to effective gene therapy of haemophilia B with AAV serotype 2-based vectors following administration of the vector in the systemic circulation.

\section{The rationale for our gene therapy strategy for haemophilia B}

We developed an approach for gene therapy of haemophilia B with the aim of overcoming some of the obstacles faced by the previous AAV-based haemophilia B clinical trials. A codon-optimised version of the human $F I X(h$ FIX co) gene was synthesised and cloned downstream of a compact synthetic liver-specific promoter $(L P l)$ to enable packaging into self-complementary AAV vectors (scAAVLP1-hFIXco), which have a packaging capacity of only $2.3 \mathrm{~kb}$ [13]. Our preclinical studies in mice and non-human primates (NHP) showed that scAAV vectors were more potent than comparable single-stranded AAV (ssAAV) vectors, raising the possibility of achieving therapeutic levels of hFIX using lower and potentially safer doses of vector [13, 14].

Another critical aspect of our study was the use of vectors pseudotyped with AAV serotype 8 capsid. This offered several potential advantages over strategies using AAV2 vectors. In particular, the remarkable tropism of AAV8 was exploited to enable efficient transduction of the liver following administration of the vector in the peripheral circulation [14, 15]. This simple non-invasive route of vector administration made vector administration safer for patients with a bleeding diathesis than when vector was administered using more invasive procedures. Additionally, the lower seroprevalence of AAV8 in humans ( $25 \%$ compared with over $70 \%$ for AAV2 [16]), enabled us to exclude subjects with pre-existing humoral immunity to AAV8 from participating in the trial, thus reducing the risk of gene transfer of the liver being blocked by the presence of neutralising anti-AAV8 antibodies.

The safety and efficacy of peripheral vein administration of scAAV-LP1-hFIXco was assessed in NHP prior to initiating the clinical trial. Equivalent levels of transgene expression at approximately $20 \%$ of normal levels were observed after peripheral vein and liver targeted delivery of scAAV. Expression remained stable over a period of $>5$ years after a single bolus administration of scAAV vector. Importantly, the biodistribution of scAAV2/8-LP1hFIXco following peripheral vein administration was comparable to that observed after administration of the same dose of vector into the mesenteric vein [17]. Notably, successful transduction was achieved in macaques with pre-existing immunity to AAV resulting from natural infection with wild-type virus after peripheral venous 
administration of vectors based on alternative serotypes of AAV. This suggested that there was little immune crossreactivity between AAV serotypes in non-humanprimates. Molecular studies confirmed that most of the AAV genomes were maintained in an episomal form [13, 17]. However, integration of vector into the host cell genome was detected at a low frequency and seemed to occur randomly within coding and non-coding regions with no evidence of activation of oncogenes or disruption of tumour suppressor genes (unpublished data). Consistent with this, liver tumours were not observed following gene transfer with scAAV2/8-LP1-hFIXco in NHP $(n=23)$ as assessed by annual ultrasound scans and regular survey of the liver following laparotomy for serial liver biopsies. Thus, in NHP primates, peripheral vein administration of scAAV resulted in long-term expression of human FIX at therapeutic levels without any toxicity, supporting the cautious extension of this approach to human subjects.

\section{The enrolment process for the Phase I trial of scAAV2/8-LP1-hFIXco}

Enrolment was restricted to subjects over the age of 18 years with severe haemophilia B (FIX $<1 \%$ of normal) with no evidence of FIX inhibitors, negative for hepatitis C RNA and HIV, and with normal liver function [18]. An elaborate two-stage informed consent process was introduced to ensure that the subjects interested in the gene therapy trial understood the risks of participating in thisthe first study-to use scAAV pseudotyped vectors encoding a codon-optimised FIX cDNA in humans. At the first level (screening consent), the trial process was outlined and likely risks explained so that informed consent for tests to determine eligibility could be given. Common reasons for "screen failures" included the presence of preexisting antibodies to AAV serotype 8, evidence of ongoing infection with hepatitis $\mathrm{B}$ or $\mathrm{C}$ virus or $\mathrm{HIV}$, or a higher risk of developing neutralising antibody to FIX protein based on genotype analysis. A secondlevel of more detailed informed consent was carried out for the subjects eligible to proceed, with independent assessment of the subjects' grasp of the risk involved and willingness to enter the trial by an ombudsman.

\section{The main observations of the Phase I clinical trial}

The first 6 patients to enter the trial were recruited in London. These individuals were divided into 3 cohorts of 2 participants each and received scAAV2/8-LP1-hFIXco vector by peripheral vein at vector doses of $2 \times 10^{11}$, $6 \times 10^{11}$ and $2 \times 10^{12} \mathrm{vg} / \mathrm{kg}$.
The low-dose subjects who consented did so in the full knowledge that the vector dose they would receive was unlikely to benefit them, and would also preclude their having a further dose of the same vector as their immune system would reject subsequently administered AAV particles of the same serotype. Both low-dose subjects achieved FIX levels of between 1 and $2 \%$ of normal as assessed by a bioactivity assay (one-stage clotting assay) following vector infusion [18]. This level was significantly higher than their baseline FIX activity of $<1 \%$ of normal and was consistent with transgene-mediated synthesis of FIX. The first subject was able to discontinue prophylaxis and has remained free of spontaneous bleeds, though he has required on-demand FIX treatment on 8 occasions over a period of 2 years to cover accidental injuries and elective surgery. The second patient has needed to continue on prophylaxis, albeit with a decreased frequency of dosing, due to much more severe pre-existing joint damage which further increased his risk of bleeding.

As both subjects had attained levels of less than $3 \%$, which was below our therapeutic endpoint, the next two subjects were treated at the intermediate dose level of $6 \times 10^{11} \mathrm{vg} / \mathrm{kg}$. Subject 3 attained a stable base line of $2 \%$, but due to pre-existing joint damage has needed to continue prophylaxis, but at an interval of once every 2 weeks compared to twice weekly before gene transfer. Subject 4 attained a baseline level of approximately $2 \%$ and has not required prophylaxis following gene transfer.

At the highest dose level of $2 \times 10^{12} \mathrm{vg} / \mathrm{kg}$, peak hFIX levels of 8 and $12 \%$ were achieved in subjects 5 and 6 , respectively. Subject 5 appeared to develop a cellular immune response to transduced hepatocytes, which resulted in a tenfold increase in liver enzymes and a drop in hFIX levels to $3 \%$ of normal at around 7 weeks after therapy. A course of prednisolone was administered, leading to resolution of liver inflammation without complete loss of transgene expression. This subject had not required prophylaxis for over 12 months following gene transfer, but has subsequently been commenced on a once a week regimen to avert trauma-related bleed incurred in the course of his work as a geologist. Subject 6 also developed a slight increase in liver enzyme levels over baseline at around 9 weeks following therapy. Although the liver enzyme levels were still within the normal range, the subject was started on a course of prednisolone early to avert a progressive $\mathrm{T}$ cell response against the transduced hepatocytes. His liver enzyme levels promptly returned to baseline values and he has not required any treatment with FIX concentrates despite living a very active life, which involves playing soccer and running in marathons on a regular basis.

None of the subjects developed neutralising antibodies to hFIX, though all 6 participants did develop a humoral 
immune response to AAV8 capsid, as expected. More crucial was the development of a $\mathrm{T}$ cell-mediated immune response to the viral capsid, as assessed by ELISpot assay. This response appeared to be dose dependent, as it did not appear in the low-dose cohort, but was detectable above baseline values in the subjects in the intermediate and highdose cohorts. It is unclear why transaminitis was not observed in the intermediate dose cohort. One possibility is that the capsid antigen load in these subjects may have been lower than the threshold required to trigger a "fullblown" immunological response resulting in loss of hepatocytes and a rise in liver enzymes.

In sum, our novel approach with scAAV vectors shows promise in subjects with severe haemophilia B because of its potential to ameliorate the bleeding diathesis through continuous expression of human FIX, after a single administration of a vector without long-lasting toxicity.

\section{Advancing beyond the first clinical success for haemophilia $B$}

Our study, though successful, raised important questions about liver inflammation, which was not observed in animal models, including non-human primates, despite administration of doses that were at least tenfold higher than the highest dose administered in humans [17]. Therefore, to better understand the safety and efficacy profile of scAAV2/8-LP1-hFIXco, we have recently enrolled four more severe haemophilia B subjects at the high-dose level with the aim of establishing: (1) if perturbation of liver enzymes occurs in all subjects treated at the high-dose level; (2) the precise time point after gene transfer when an increase in liver enzymes occurs to enable targeted use of immunomodulators; and (3) if early institution of prednisolone diminishes the risk and/or magnitude of hepatocellular toxicity whilst preserving transgene expression. The outcomes in these four new subjects will be reported once we have accrued sufficient safetydata.

A way of reducing the risk of liver inflammation is to reduce the total capsid load infused into subjects at the high-dose level. The vector preparation used in our study contained a large excess $(\sim 80 \%)$ of empty capsids which are fully assembled capsids that lack a functional viral genome [19]. These empty particles cannot mediate FIX expression, but can serve as antigenic targets for capsidspecific cytotoxic $\mathrm{T}$ cells following transduction of hepatocytes [20]. Since the dose administered to humans is based on an estimate of the proviral genome copy number, and not the total viral capsid load, it seems logical to assume that removal of these contaminating empty particles may allow administration of the high vector dose without provoking hepatocellular toxicity or compromising the level of gene transfer. To test this hypothesis further, we have prepared another clinical lot ofscAAV2/8-LP1hFIXco from which empty particles have been removed by $\mathrm{CsCl}$ density centrifugation. We plan to infuse this new vector preparation in subjects with severe haemophilia B early in 2014.

Another strategy for reducing vector-related toxicity currently being investigated in the clinic entails the use of a self-complementary AAV vectors encoding FIX Padua, a variant with a single amino acid change of an arginine at position 338 in the catalytic domain of FIX to leucine. This results in an almost tenfold higher specific activity in the context of gene transfer to the liver or the muscle. This gain of function mutant, therefore, raises the possibility of correcting the severe bleeding phenotype in haemophilia B with a lower vector dose. A cautious evaluation of this approach is underway in the United States [21, 22].

Dr Kathy High's group at the Children's Hospital of Philadelphia is exploring a novel approach that has the potential to overcome pre-existing neutralising antibodies to AAV. They showed in preclinical settings that a noninfectious, low immunogenic AAV mutant derived from AAV2 can be used as decoy to effectively adsorb preexisting neutralising antibodies in a dose-dependent manner, thus overcoming their inhibitory effect and maximising gene transfer [23]. Therefore, by optimising the ratio of full/empty capsids in the final formulation of vector, based on a patient's anti-AAV titres, it may be possible to enrol patients currently excluded from participating in gene therapy clinical trials because of the presence of preexisting neutralising anti-AAV antibodies. A clinical trial assessing this approach is underway and has enrolled two subjects.

\section{Summary}

Until 50 years ago, the treatment of haemophilia was virtually limited to the infusion of fresh frozen plasma. In 1968 the first widely available concentrate for haemophilia A-cryoprecipitate was introduced. During the 1970s and 1980s many multidonor factor concentrates were developed, but this led directly to the HIV and hepatitis C catastrophe, resulting in the loss of a generation of haemophiliacs. Now there are safe (but expensive) recombinant factors. In the future we can look forward to gene therapy, as it is now possible to achieve stable expression of FIX at therapeutic levels following a single administration of AAV vector without any persistent safety concerns. However, longer follow-up of a larger cohort of patients is essential to establish the duration of transgene expression and the absence of long-term toxicity following gene transfer. 


\section{References}

1. Nathwani AC, Tuddenham EG. Epidemiology of coagulation disorders. Baillieres Clin Haematol. 1992;5:383-439.

2. Ponder KP, Srivastava A. Walk a mile in the moccasins of people with haemophilia. Haemophilia. 2008;14:618-20.

3. Pipe SW. Hemophilia: new protein therapeutics. Hematology Am Soc Hematol Educ Program. 2010;2010:203-9.

4. Pipe SW. The hope and reality of long-acting hemophilia products. Am J Hematol. 2012;87(Suppl 1):S33-9.

5. Nathwani AC, Davidoff AM, Tuddenham EG. Prospects for gene therapy of haemophilia. Haemophilia. 2004;10:309-18.

6. Nakai $\mathrm{H}, \mathrm{Wu} \mathrm{X}$, Fuess S, et al. Large-scale molecular characterization of adeno-associated virus vector integration in mouse liver. J Virol. 2005;79:3606-14.

7. Herzog RW, Hagstrom JN, Kung SH, et al. Stable gene transfer and expression of human blood coagulation factor IX after intramuscular injection of recombinant adeno-associated virus. Proc Natl Acad Sci USA. 1997;94:5804-9.

8. Herzog RW, Yang EY, Couto LB, et al. Long-term correction of canine hemophilia B by gene transfer of blood coagulation factor IX mediated by adeno-associated viral vector [see comments]. Nat Med. 1999;5:56-63.

9. Snyder RO, Miao CH, Patijn GA, et al. Persistent and therapeutic concentrations of human factor IX in mice after hepatic gene transfer of recombinant AAV vectors. Nat Genet. 1997;16:270-6.

10. Manno CS, Chew AJ, Hutchison S, et al. AAV-mediated factor IX gene transfer to skeletal muscle in patients with severe hemophilia B. Blood. 2003;101:2963-72.

11. Jiang H, Pierce GF, Ozelo MC, et al. Evidence of multiyear factor IX expression by AAV-mediated gene transfer to skeletal muscle in an individual with severe hemophilia B. Mol Ther. 2006;14: $452-5$.

12. Manno CS, Arruda VR, Pierce GF, et al. Successful transduction of liver in hemophilia by AAV-Factor IX and limitations imposed by the host immune response. Nat Med. 2006;12(3):342-7.

13. Nathwani AC, Gray JT, Ng CY, et al. Self complementary adenoassociated virus vectors containing a novel liver-specific human factor IX expression cassette enable highly efficient transduction of murine and nonhuman primate liver. Blood. 2006;107: 2653-61.

14. Nathwani AC, Gray JT, McIntosh J, et al. Safe and efficient transduction of the liver after peripheral vein infusion of self complementary AAV vector results in stable therapeutic expression of human FIX in nonhuman primates. Blood. 2007;109:1414-21.

15. Thomas CE, Storm TA, Huang Z, Kay MA. Rapid uncoating of vector genomes is the key to efficient liver transduction with pseudotyped adeno-associated virus vectors. J Virol. 2004;78: 3110-22.

16. Gao GP, Alvira MR, Wang L, et al. Novel adeno-associated viruses from rhesus monkeys as vectors for human gene therapy. Proc Natl Acad Sci USA. 2002;99:11854-9.

17. Nathwani AC, Rosales C, McIntosh J, et al. Long-term safety and efficacy following systemic administration of a self-complementary AAV vector encoding human FIX pseudotyped with serotype 5 and 8 capsid proteins. Mol Ther. 2011;19:876-85.

18. Nathwani AC, Tuddenham EG, Rangarajan S, et al. Adenovirusassociated virus vector-mediated gene transfer in hemophilia B. N Engl J Med. 2011;365:2357-65.

19. Allay JA, Sleep S, Long S, et al. Good manufacturing practice production of self-complementary serotype 8 adeno-associated viral vector for a hemophilia B clinical trial. Hum Gene Ther. 2011;22:595-604.

20. Pien GC, Basner-Tschakarjan E, Hui DJ, et al. Capsid antigen presentation flags human hepatocytes for destruction after transduction by adeno-associated viral vectors. J. Clin. Invest. 2009; 119:1688-95.

21. Simioni P, Tormene D, Tognin G, et al. X-linked thrombophilia with a mutant factor IX (factor IX Padua). $\mathrm{N}$ Engl J Med. 2009;361:1671-5.

22. Finn JD, Nichols TC, Svoronos N, et al. The efficacy and the risk of immunogenicity of FIX Padua (R338L) in hemophilia B dogs treated by AAV muscle gene therapy. Blood. 2012;120:4521-3.

23. Mingozzi F, Anguela XM, Pavani G et al. Overcoming preexisting humoral immunity to AAV using capsid decoys. Sci Transl Med. 2013;5:194ra92. 